\title{
Could be considered serum anti-S-100 autoantibodies a biomarker in patients with acute non-cardioembolic ischemic stroke?
}

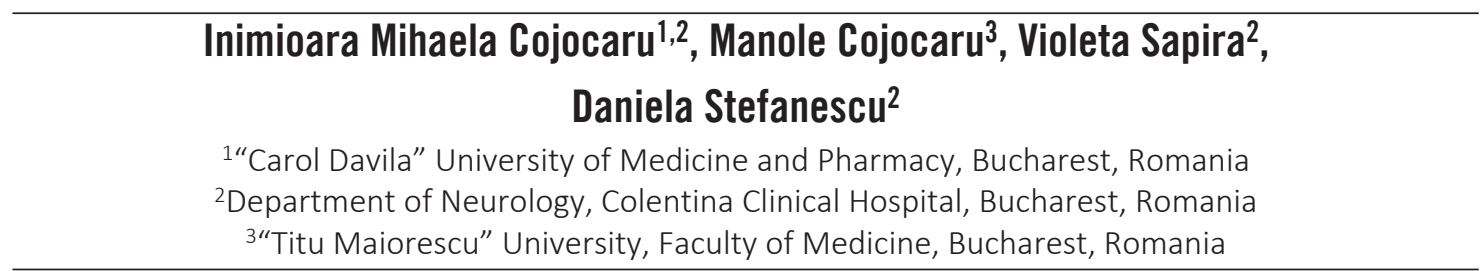

\begin{abstract}
In ischemic stroke patients there were found autoantibodies against brain epitopes. The presence of anti-S-100 autoantibodies may reflect alteration and dysfunction of the blood-brain barrier. The aim of the study was to assess the prevalence of serum anti-S-100 autoantibodies in the early stage of ischemic non-cardioembolic stroke and their correlation with clinical outcome. The study included 78 patients with acute non-cardioembolic ischemic stroke, 46 females and 32 males, ranges from 46 to 81 years, mean age $74.3 \pm 6.8$ years. Blood samples for anti-S-100 autoantibodies measurement were taken within 24 hours after the onset of ischemic stroke. Seventy-two patients served as matched controls 43 females and 29 males, ranges from 42 to 79 years, mean age $71.3 \pm 6.4$ years. Serum anti-S-100 autoantibodies were measured by means of ELISA. Significance difference between groups was calculated by the Wilcoxon signed rank method. A value of $p<0.05$ was considered significant. Anti-S-100 autoantibodies were detected in 25 patients (32\%). Patients with acute ischemic stroke had significantly higher levels of anti-S-100 autoantibodies $(87.3 \pm 9.23 \mathrm{IU} / \mathrm{ml})$ as compared to healthy controls $(23.1 \pm 1.17 \mathrm{IU} / \mathrm{ml})(p<0.01)$. In 24 patients the cerebral CT scan was negative in the first 24 hours, out of them in 6 patients anti-S-100 autoantibodies were positive. The higher titers correlated significantly with worse clinical outcome $(132.0 \pm 12.4 \mathrm{IU} / \mathrm{ml} ; \mathrm{p}<0.001)$. The negative predictive value of anti-S-100 autoantibodies was $87 \%$, and positive predictive value of anti-S-100 autoantibodies was $71 \%$. Measurement of anti-S-100 autoantibodies may be used to select patients with early negative cerebral CT scanning as presenting ischemic stroke.
\end{abstract}

Keywords: anti-S-100 autoantibodies, acute non-cardioembolic ischemic stroke, prevalence

\section{INTRODUCTION}

Stroke is the leading cause of severe long-term disability and the third leading cause of death worldwide. Acute ischemic stroke is caused by thrombotic or embolic occlusion of a cerebral artery $(1,2)$.

Acute ischemic stroke often can be reversed successfully with the clot lysis drug recombinant tissue plasminogen activator (rt-PA), if initial evaluation is immediate and intravenous infusion begins within 3 hours of stroke symptom onset (3).

After stroke, there is a breakdown of the bloodbrain barrier that allows for an encounter of central nervous system (CNS) antigens by the systemic immune system; this encounter can occur in the injured brain as well as in the periphery $(4,5)$.

Immune responses to brain antigens occur after stroke, and experimental studies show that the likelihood of developing a detrimental autoimmune response to these antigens is increased by systemic inflammation at the time of stroke (6-8).

Our objectives were to study the prevalence serum anti-S-100 autoantibodies in patients with acute ischemic stroke and to evaluate their prognostic significance. 


\section{MATERIAL AND METHODS}

Sera from subjects with different subtypes of acute non-cardioembolic ischemic stroke as TOAST and OCSP criteria within 24 hours after stroke onset were screened for reactivity against S-100 proteins $(1,2,10)$.

Seventy-eight patients (ranges from 46 to 81 years, mean age $74.3 \pm 6.8$ years, M/F: $32 / 46$ ) with acute ischemic stroke were recruited from those admitted in the Neurological Intensive Care Unit (NICU). In the academic medical center emergency department, as the patient arrives, a suspected stroke is treated as an acute event until diagnostic evidence suggests otherwise.

National Institutes of Health Stroke Scale (NIHSS) and Glasgow Outcome Score (GOS) at baseline were used $(9,12)$.

In addition one control group consisting of 72 healthy individuals (ranges from 42 to 79 years, mean age $71.3 \pm 6.4$ years, M/F: 29/43) were recruited.

Exclusion criteria from the study were: history of atrial fibrillation, valvular heart disese, (as shown on echocardiography), as were those with a previously undetected cardiac source of thrombus identified by clinical assessment, non-cortical stroke, subarachnoid hemorrhage, connective tissue disease, severe medical or psychiatric illness, inflammatory gastrointestinal disease, serum creatinine $>150 \mathrm{mmol} / \mathrm{l}$, gout or renal failure or took of antioxidant vitamins, iron deficiency.

According to the standard protocol, physicians in the NICU recorded clinical data from the primary evaluation, including history and examination results.

Cerebral ischemia was documented by an imaging technique, such as computerized tomography (CT scan) or magnetic resonance imaging (MRI).
Ultrasound examination of cervico-cerebral arteries and of heart was performed.

Local ethics committees of both the University and Institute approved the clinical protocols, and all patients, including controls, provided informed consent.

A sample $(5 \mathrm{ml})$ was taken within 24 hours after the onset of ischemic stroke by venipuncture into vacuum tubes without anticoagulant (Becton Dickinson), and serum was separated by centrifugation at $4,000 \mathrm{~g}$ for $5 \mathrm{~min}$ at $4^{\circ} \mathrm{C}$ and stored as $0.5 \mathrm{ml}$ aliquots at $-80^{\circ} \mathrm{C}$ until an ELISA was performed, according to the manufacturer's instruction manual. Serum anti-S-100 autoantibodies were measured by means of ELISA.

The results were expressed as arbitrary units (IU/ml) by reading of a standard curve. Mean values (x) \pm standard deviation (SD) and 95\% confidence intervals (CIs) were used. A ROC curve was used to calculate the cutoff value for optimal sensitivity and specificity. Significance differences between groups was calculated by the Wilcoxon signed rank method. Titers of anti-S-100 autoantibodies were compared by the Student $t$ test. A value of $p<0.05$ was considered significant.

\section{RESULTS}

Anti-S-100 autoantibodies was detected in 25 (32\%) patients with non-cardioembolic ischemic stroke.

Patients with acute ischemic stroke had significantly higher levels of anti-S-100 autoantibodies $(87.3 \pm 9.2 \mathrm{IU} / \mathrm{ml})$ as compared to healthy controls $(23.1 \pm 1.17 \mathrm{IU} / \mathrm{ml})(\mathrm{p}<0.001)($ Fig. 1).

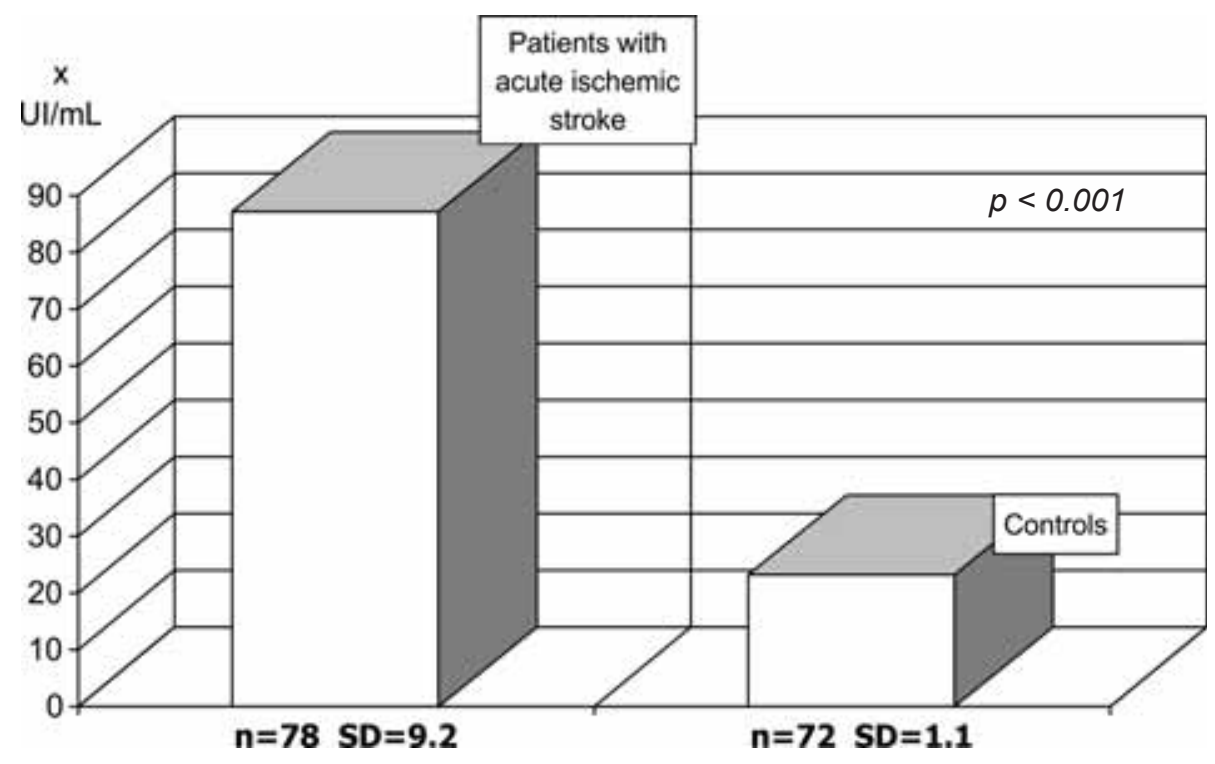

FIGURE 1. Mean value of anti-S100 autoantibody titers in patients with acute non-cardioembolic ischemic stroke as compared to controls 
In 24 patients the cerebral CT scan was negative in the first 24 hours, out of them in 6 patients anti-S-100 autoantibodies were positive.

A significant statistical correlation between anti-S-100 autoantibody titers and GOS $(r=0.71$, $\mathrm{p}<0.018$ ) and a significant statistical correlation between anti-S-100 autoantibody levels of NIHSS groups (16-42 points-bad prognosis, 7-15 points-intermediate prognosis, and 0-6 points-good prognosis) were observed $(r=0.76, p<0.003)$.

The negative predictive value of anti-S-100 autoantibodies was $87 \%$, and positive predictive value of anti-S-100 autoantibodies was $71 \%$.

The results confirm that these autoantibodies are released into the blood after stroke, they can be easily measured, and they correlate with outcome. The titer correlated significantly with clinical outcome $(132.0 \pm 12.4 \mathrm{IU} / \mathrm{ml} ; \mathrm{p}<0.001)$. Higher peak of serum anti-S-100 autoantibodies levels correlated well with worse clinical outcome. From these results a relationship between autoantibody titers and ischemic stroke seems possible.

\section{DISCUSSION}

Stroke is defined as an acute neurologic dysfunction of vascular origin with sudden (within seconds) or at least rapid (within hours) occurrence of symptoms and signs corresponding to the involvement of focal areas in the brain.

Some studies demonstrate that immune responses to brain antigens occur after stroke, although these responses are likely to be an epiphenomenon of ischemic brain injury $(6,7,12)$.

Endothelial cells, pericytes, basal lamina and astrocytes are the constitutive elements of the BBB and serve to protect the brain from sudden changes of plasma composition and to regulate exchanges between parenchymal and vascular compartiments (13).

Because the BBB is disrupted and presents increased permeability after ischemic stroke $(14,15)$ lymphocytes infiltrate the ischemic brain allowing for contact with CNS antigens, in both the brain and periphery from numerous different cell types (neurons, astrocytes, oligodendrocytes), which are normally sequestered from the peripheral immune system, allowing for the development of a CNS antigen-specific autoimmune response (16).

Furthermore, there is an increase in the concentration of antigens such as myelin basic protein (MBP), neuron specific endolase (NSE), S-100 proteins, and glial fibrillary acidic protein (GFAP) in the systemic circulation after stroke, which al- lows for lymphocyte encounter with these antigens in peripheral lymphoid organs. Given this lymphocyte contact with novel CNS antigens, irrespective of site, it is thus possible for an (auto)immune response to occur to these antigens. Indeed, antibodies and cellular immune responses to brain antigens are documented in stroke survivors.

Presence of S-100 in serum after ischemic stroke may be due to combined leakage out of necrotic glial cells and passage through an impaired blood-brain-barrier, indicating severe ischemic cell injury $(17,18)$.

The degree of systemic inflammation following stroke correlates with stroke severity and to the amount of tissue infarcted; the amount of tissue infarcted is also reflected by the concentration of the above mentioned antigens (19).

At higher concentrations, S-100b has been shown to stimulate proinflammatory cytokine production and apoptosis in vitro (20).

The anti-S-100 antibodies react with alpha and beta subunits of human S-100 proteins (21-24 kD). S-100 proteins are low-molecular-weight calcium binding proteins that are believed to play an important role in various cellular processes such as regulation of protein phosphorilation, cell growth and motility, cell cycle, transcription, differentiation and cell survival, acting intracellulary or extracellulary (21).

In the nervous system, S-100b, the beta-beta homodimer, is concentrated in astrocytes and other glial cell types, such as oligodendrocytes, Schwann cells, ependymal cells, enteric glial cells, retinal Muller cells, and in definite neuron subpopulations. In non-neural tissues, the protein is widely distributed in definite cell types, including melanocytes, Langerhans cells, chondrocytes, dendritic cells, Leydig cells, skeletal muscle satellite cells, adipose tissue (21-23).

Both blood and CSF S-100b levels have been shown to be reliable biomarkers to predict outcomes in various clinical conditions, when measured against functional scores, symptom inventories, or measures of neuropsychological impairment in both brain trauma and brain insults associated with cardiovascular disorders $(24,25)$.

Early neurological deterioration, defined as worsening of neurological status from admission to 48-72 hours after admission, is associated also with high S-100b level (26).

A direct involvement of S-100b overproduction as a sign of maladaptative astrocytic activation was observed in pathogenic mechanism accompanying brain ischemia $(27,28)$. 
The increase of S-100b in serum after acute ischemic stroke peaks at 2-3 days, and it can be observed at approx. $12 \mathrm{~h}$, it predicts a malignant course of infarction in the proximal middle cerebral artery occlusion (29).

Some studies have concluded also that measuring blood concentration of S-100 protein periodically in the first 10 days after cerebral infarction help to predict infarct volume and the long-term neurological outcome (30).

Some authors have found a transient increase of S-100 protein in the CSF during the first week after the ischemic stroke, which was significantly correlated with the size of the infarction and the clinical

In patients with ischemic stroke it was observed a strong correlation between the values of C-reactive protein, marker of inflammation, and those of $\mathrm{S}-100 \mathrm{~b}$, independently of the size of infarction (32).

The enhanced synthesis of S-100b by reactive astrocytes participate in the inflammatory response within the periinfarct area, and may be related to the occurrence of delayed expansion and prolonged neurologic deficits (33).

Serum S-100 protein is considered a marker of cerebral injury after cardiac surgery performed under cardiopulmonary bypass (34).

Other authors have observed that serum S-100 protein was a valid and reliable predictor of the outcome at 24 hours after acute global cerebral ischemia $(35,36)$.

S-100 protein levels were observed significantly higher in patiens with acute cardioembolic and atherotrombotic stroke as compared to transient ischemic attack (TIA) group and they were significantly higher in the atherotrombotic stroke group than in the lacunar stroke and TIA groups, and in the cardioembolic stroke group compared to lacunar stroke group. S-100 protein levels were significantly higher in patients who died and they were indicators for predicting stroke subtypes and severity (37).

The expression of S-100 proteins have been evaluated in a variety of disorders, for example, as serum markers for subjects with inflammatory disstate of the patients (31).

eases. Dosage of anti-S-100 autoantibodies does not appear useful for diagnostic purpose because of the overlap observed among groups $(8,38)$.

The immune response to CNS antigens like S-100 protein that occur after acute ischemic stroke is not novel, but the finding that these responses may have pathological consequences is, as we have observed in a previous paper (39).

Measurement of anti-S-100 autoantibodies may be used to select patients with negative early cerebral CT scanning as presenting ischemic stroke.

The obtained data allowed us to consider serum level of autoantibodies to S-100 proteins as a marker of ischemic brain lesion, and to suppose further potential role of this autoantibodies in cerebrovascular disease progression.

Further research would be necessary to confirm if antibodies to S-100 proteins could be of clinical importance in acute ischemic stroke.

\section{CONCLUSIONS}

Elevated levels of circulating autoantibodies reactive against several S-100 proteins were detected in the sera of non-cardioembolic ischemic stroke.

The presence of anti-S-100 autoantibodies seems to reflect an alteration of the blood-brain barrier that promotes the access of central nervous system antigens to immunocompetent cells.

From these results a relationship between autoantibody titers and stroke seems possible.

The detection of increased serum levels of autoantibodies to S-100 proteins constitutes a novel strategy for selecting patients with negative early cerebral CT scanning as presenting ischemic stroke and for prognosis of ischemic stroke.

Autoantibodies anti-S-100 proteins may be considered a biomarker of acute ischemic stroke and their presence suggests the involvement in cerebral infarct progression.

Significant statistical correlations between anti-S-100 antibody titers and clinical outcome were observed (NIHSS ans GCS score)

\section{Acknowledgements}

These authors contributed equally to this work.

Conflict of interest: none declared

\section{REFERENCES}

1. Adams H.P.J., Bendixen B.H., Kapello I.J. Biller J., Love B. B., Gordon D.L. et al. Classification of subtype of acute ischemic stroke. Definition of use in a multicentric trial/TOAST. Trial of Org 10172 in Acute Stroke Treatment. Stroke 1993; 24: 35.
2. Pittock S.J., Meldrum D., Hardiman O., Thornton J., Brennan P., Moroney J.T. The Oxfordshire Community Stroke Project classification: correlation with imaging, associated complications, and prediction of outcome in acute ischemic stroke. J Stroke Cerebrovasc Dis. 2003; 12: 1-7. 
3. Jauch E.C., Saver J.L., Adams H.P.Jr., Bruno A., Connors J.J. (Buddy), Demaerschalk B.M. et al. Guidelines for the early management of patients with ischemic stroke: A guideline for healthcare professionals from the American Heart Association/ American Stroke Association. Stroke. 2013; 44: 870-947.

4. Hill M.D. Diagnostic biomarkers for stroke: a stroke neurologist's perspective. Clin. Chem. 2005; 51(11): 2001-2002.

5. Whiteley W., Tseng M.C., Sandercock P. Blood biomarkers in the diagnosis of ischemic stroke: a systematic review. Stroke. 2008; 39(10): 2902-2909.

6. Bornstein N.M., Aronovich B., Korczyn A.D., Shavit S., Michaelson D.M., Chapman J. Antibodies to brain antigens following stroke. Neurology. 2001; 56: 529-530.

7. Zierath D., Thullbery M., Hadwin J., Gee J.M., Savos A., Kalil A. et al. CNS immune responses following experimental stroke. Neurocrit Care. 2010; 12: 274-284.

8. Mecocci P., Parnetti L., Romano G., Scarelli A., Chionne F., Cecchetti R. et al. Serum anti-GFAP and anti-S100 autoantibodies in brain aging, Alzheimer's disease and vascular dementia. $J$ Neuroimmunol. 1995; 57: 165-170.

9. Adams H.P.Jr., Davis P.H., Leira E.C., Chang K.C., Bendixen B.H., Clarke W.R. et al. Baseline NIH Stroke Scale score strongly predicts outcome after stroke. A report of the Trial of Org 10172 in Acute Stroke Treatment (TOAST) Neurology 1999; 53: 125-131.

10. Lynch J.R., Blessing R., White W.D., Grocott H.P., Newmann M.F., Laskowitz D.T. Novel diagnostic test for acute stroke. Stroke 2004; 35: 57-63.

11. Adams H.P.JR., Leclerc J.R., Bluhmki E., Clarke W., Hansen M.D., Hacke W. Measuring outcomes as a function of baseline severity of ischemic stroke. Cerebrovascular Dis 2004; 18: 124-129.

12. Becker K.J. Modulation of the postischemic immune response to improve stroke outcome. Stroke 2010; 41[suppl 1]: S75-S78.

13. Engelhardt B. Development of the blood-brain barrier. Cell Tissue Res 2003; 314: 119-129.

14. Hilario E., Rey M.C., Goni F., Alvarez F.J., Caballero A., Gastiasoro E. et al. Cerebral blood flow and morphological changes after hypoxic-ischemic injury in preterm lambs. Acta Pediatr 2006; 94: 903-911

15. Goni de-Cerio F., Alvarez A., Alvarez F.J., Rey-Santano M.C., Alonso-Alconada D., Mielgo V.E. et al. $\mathrm{MgSO}_{4}$ treatment preserves the ischemia-induced reduction in S-100 protein without modifications of the expression of endothelial tight junction molecules. Histol Histopathol 2009: 24: 1129-1138.

16. Gee J.M., Kalil A., Shea C., Becker K.J. Lymphocytes. Potential mediators of postischemic injury and neuroprotection. Stroke 2007; 38[part 2]: 783-788.

17. Bütner T., Weyers S., Postert T., Sprengelmeyer R., Kuhn W. S-100 protein: serum marker of focal brain damage after ischemic territorial MCA infarction. Stroke 1997; 28: 1961-1967

18. Sotgiu S., Zanda B., Marchetti B., Fois M.L., Amu G., Pes G.M. et al. Inflammatory biomarkers in blood of patients with acute brain ischemia. Eur. J. Neurol 2006; 13(5): 505-513.

19. Jauch E.C., Lindsell C., Broderick J., Fagan S.C., Tilley B.C., Levine S.R. Associations of serial biochemical markers with acute ischemic stroke: the National Institute of Neurological Disorders and Stroke Recombinant Tissue Plasminogen Activator Stroke Study. Stroke 2006; 37; 2508-2513.

20. Maas M., Furie K.L. Molecular biomarkers in stroke and prognosis. Biomark Med 2009; 3: 363-383

21. Michetti F., Corvino V., Geloso M.C., Lattanzi W., Bernardini C., Serpero L. et al. The S100B protein in biological fluids: more than a lifelong biomarker of brain distress. J. Neurochem 2012; 120: 644-659.

22. Laterza O., Modur V.R., Crimmins D.L., Olander J.V., Landt Y., Lee J.M. et al. Identification of novel brain biomarkers. Clinical Chemistry 2006; 52: 1713-1721.

23. Tubaro C., Arcuri C., Giambanco L., Donato R. S100B protein in myoblasts modulates myogenic differentiation via NF-kappa-beta- dependent inhibition of MyoD expression. J Cell Physiol 2010; 223: 270-282.

24. Foerch C.M., Singer O.C., Neumann-Haefelin T., Du Mesnil de Rochemont R., Steinmetz H., Sitzer M. et al. Evaluation of serum $\mathrm{S} 100 \mathrm{~b}$ as a surrogate marker for long-terme outcome and infarct volume in acute middle cerebral artery infarction. Arch Neurol 2015; 62: $1130-1134$.

25. Sanchez-Perez P., Pereira A.R., Sourcer N.A., Biondi A., Lejean L., Colonne C. et al. S100b as an additional prognostic marker in subarachnoid aneurismal hemorrhage. Crit. Care Med 2008; 36: 2267-2273.

26. Jickling G.C, Sharp F.R. Blood biomarkers of ischemic stroke. Neurotherapeutics 2011; 8: 349-360.

27. Assano T., Mori T., Shimoda T., Shinagawa R., Satoh S., Yada N. et al. Arundic acid (ONO-2506) ameliorates delayed ischemic brain damage by preventing astrocytic overproduction of S100b. Curr Drug Targets CNS Neurol Disord 2005; 4: 127-142.

28. Mori T., Tan J., Arendash G.W., Koyama N., Nojima Y., Town T. Overexpression of human $\mathrm{S} 100 \mathrm{~b}$ exacerbates brain damage and periinfarct gliosis after permanent focal ischemia. Stroke 2008; 39: 2114-2121.

29. Foerch C., Otto B., Singer O.C., Neumann-Haefelin T., Yan B., Berkefeld J. et al. Serum S100b predicts a malignant course of infarction in patients with acute middle cerebral artery occlusion. Stroke 2004; 35: 2160-2164.

30. Missler U., Wiesman M., Friedrich C., Kaps M. S100 protein and neuron-specific enolase concentrations in blood as indicators of infarction volume and prognosis in acute ischemic stroke. Stroke 1997; 28: 1956-1960.

31. Aurell A., Rosengren L.E., Karlsson B., Olsson J.E., Zbornikova V., Haglid K.G. Determination of S-100 and glial fibrillary acidic protein concentrations in cerebrospinal fluid after brain infarction. Stroke 1991; 22: 1254-1258.

32. Beer C., Blacker D., Bynevelt M., Hankey G.J., Puddley I.B., Systemic markers of inflammation are independently associated with S100b concentration: results of an observational study in subjects with acute ischemic stroke. J Neuroinflammation 2010; 7: 71-75.

33. Matsui T., Takashi M., Tateishi N., Kagamiishi Y., Satoh S., Katsube N. et al. Astrocytic activation and delayed infarct expansion after permanent focal ischemia in rats. Part I: Enhanced astrocytic synthesis of S-100b in the periinfarct area precedes delayed infarct expansion. Journal of Cerebral Blood Flow and Metabolism 2002; 22: 711-722.

34. Shaaban M.A., Harmer M., Vaughan R. Serum S100 protein as a marker of cerebral damage during cardiac surgery. British Journal of Anesthesia 2000; 85: 287-298.

35. Martens P., Raabe A., Johnsson P. Serum S-100 and neuronspecific enolase for prediction of regaining consciousness after global cerebral ischemia. Stroke 1998; 29: 2363-2366.

36. Reynolds M.A., Kirkick H.J., Dahlen J.R., Anderberg J.M., McPherson P.H., Nakamura K.K. et al. Early biomarkers of stroke. Clinical Chemistry 2003; 49: 1733-1739.

37. Üstündag M., Orak M., Güloglu C., Tamam Y., Sayhan M.B., Kale $E$. The role of serum osteoprotegerin and S-100 protein levels in patients with acute ischaemic stroke: determination of stroke subtype, severity and mortality. The Journal of International Medical Research 2011; 39: 780-789.

38. Poletaev A.B, Morozov S.G, Gnedenko B.B, Zlumkin V.M., Korzhenevsky D.A. Serum anti-S100b, anti-GFAP and anti-NGF autoantibodies of IgG class in healthy persons and patients with mental neurological disorders. Autoimmunity. 2000; 32: 33-38.

39. Cojocaru M, Cojocaru I.M., Burcin C., Atanasiu A. Determination of serum anti-S100 autoantibodies in patients with acute ischemic stroke. (Abstr.). $8^{\text {th }}$ Symposium on Autoantibodies, Dresden, 2007. 